\title{
新型含氮杂环结构的吡啶苯醚类化合物的合成与生物活性研究
}

\author{
戴红 ${ }^{a, c}$ 陈 佳 ${ }^{a}$ 曹雄飞 ${ }^{b}$ 苗文科 ${ }^{c}$ 石玉军 $*, a, b$

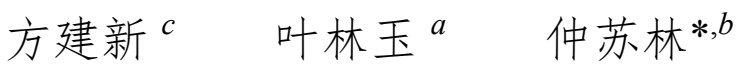 \\ ( ${ }^{a}$ 南通大学化学化工学院 南通 226019) \\ $\left({ }^{b}\right.$ 联合国南通农药剂型开发中心 南通 226006) \\ (c 南开大学元素有机化学国家重点实验室 天津 300071)
}

\begin{abstract}
摘要 为了从吡啶苯梄类衍生物中寻找新的活性化合物, 运用活性亚结构拼接原理, 设计合成了一系列未见文献报道 的新型含氮杂环结构的吡啶苯醚类化合物. 利用 ${ }^{1} \mathrm{H} N M R 、{ }^{13} \mathrm{C} N \mathrm{NR}$ 、IR 和元素分析对所制备的化合物的结构进行了 表征. 初步的生物活性测试结果表明, 部分目标化合物具有较好的杀虫活性, 部分标题化合物表现出明显的抗肿瘤活 性.
\end{abstract}

关键词 含氮杂环; 吡啶苯醚; 合成; 生物活性

\section{Synthesis and Bioactivity of Novel Pyridine Phenyl Ethers Bearing $\mathrm{N}$-heterocycles}

\author{
Dai, Hong ${ }^{a, c}$ \\ Chen, $\mathrm{Jia}^{a}$ \\ Cao, Xiongfei ${ }^{b}$ \\ Miao, Wenke ${ }^{c}$ \\ Shi, Yujun $*, a, b$ \\ Fang, Jianxin ${ }^{c}$ \\ Ye, Linyu ${ }^{a}$ \\ Zhong, Sulin ${ }^{*, b}$ \\ ( ${ }^{a}$ College of Chemistry and Chemical Engineering, Nantong University, Nantong 226019) \\ ( ${ }^{b}$ United Nations Nantong Pesticide Forulation Development Centre, Nantong 226006) \\ ( ${ }^{c}$ State Key Laboratory of Elemento-organic Chemistry, Nankai University, Tianjin 300071)
}

\begin{abstract}
In order to find novel pyridine phenyl ether lead compounds, a series of novel pyridine phenyl ether derivatives containing $\mathrm{N}$-heterocycles were prepared by the strategy of active substructure combination. Their structures were determined by ${ }^{1} \mathrm{H}$ NMR, ${ }^{13} \mathrm{C}$ NMR, IR and elemental analysis. Preliminary bioassay data displayed that some of the designed compounds exhibited good insecticidal activity. Additionally, some target compounds showed significant anti-tumor activities.
\end{abstract}

Keywords N-heterocycle; pyridine phenyl ether; synthesis; bioactivity

二芳基醚类衍生物是一类重要的有机化合物，因其 具有良好的杀菌、杀虫、除草、植物生长调节、抗肿瘤 等活性而倍受化学家与药物学家的关注, 二芳基醚类化 合物在农药、医药领域具有广泛的应用 ${ }^{[1 \sim 10]}$. 近年来不 少研究表明, 由于吡啶环的特殊效应, 用吡啶基取代二 芳基醚中的一个苯环, 往往显著改善化合物的理化性质 与生物活性. 目前含吡啶基取代的二芳醚类衍生物现已 成为新药创制研究的热点领域之一 ${ }^{[11 ~ 14]}$. 其中已商品 化的品种有吡氟禾草灵和氟吡酰草胺等 ${ }^{[15,16]}$. 此外, 2010 年冉兆晋等报道的含吡啶苯醚结构的噻二唑类化
合物对黄瓜枯萎显示出优异的杀菌作用 ${ }^{[17]} .2010$ 年申兆 晋等 ${ }^{[18]}$ 合成的含吡啶苯醚结构的丙酸酯类对马唐和苟 麻具有优异的除草作用. 新烟碱类化合物是一类重要的 乙酰胆碱酯酶受体抑制剂型杀虫剂, 其中典型性代表有 吡虫啉、氯噻啉、噻虫嗪、噻虫啉、AKD-1022 等, 因 其具有活性高、作用快、毒性低等优点, 在农业生产上

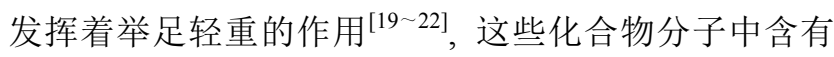
重要的 1,3-咪唑烷、1,3-噻唑烷、1,3,5-三嗪、1,3,5-惡二 嗪等杂环基团 ${ }^{[23,24]}$. 鉴于此, 为了进一步从吡啶苯醚类 化合物中寻找与发现具有较好生物活性的先导化合物,

*E-mail: yjshi2015@163.com, zhongslnt@163.com

Received October 19, 2015; revised November 5, 2015; published online November 20, 2015

Project supported by the National Natural Science Foundation of China (Nos. 21202089, 21372135), and the Science and Technology Project Fund of Nantong City (Nos. AS2013002, MS22015020, AS2014011).

国家自然科学基金(Nos. 21202089, 21372135)和南通市科技计划(Nos. AS2013004, MS22015020, AS2014011)资助项目. 
根据生物活性叠加原理, 将咪唑烷、噻唑烷、三嗪、啞 二嗪等含氮杂环结构与含吡啶基二芳醚单元有机衔接 在一起, 设计合成了一系列未见文献报道的新型含氮杂 环结构的吡啶苯醚类衍生物. 通过 ${ }^{1} \mathrm{H} N M R 、{ }^{13} \mathrm{C} \mathrm{NMR}$ 和元素分析等方法表征了所合成化合物的结构. 此外, 部分目标化合物还经 IR 进一步确证. 初步生物活性测 试结果表明, 部分目标化合物具有较好的杀虫活性, 部 分化合物表现出一定的抗肿瘤活性. 其合成路线如 Scheme 1 所示.

\section{1 结果与讨论}

\section{1 化合物的合成}

目标化合物 $\mathbf{5}$ 是通过中间体 $\mathbf{3}$ 与不同含氮杂环的缩 合反应制备而成. 以目标化合物 51 的合成为研究对象,
探讨了不同的制备条件对其反应收率的影响. 从表 1 可 以看出，选用乙腈作溶剂，碳酸钾作缚酸剂，加热回流 的方法，化合物 $5 \mathrm{I}$ 的收率为 50\%, 明显高于其它几种合 成方法的收率. 实验过程中发现该方法反应条件比较温 和，后处理简便. 最后采用该法顺利地制备出其它的目 标化合物.

表 1 不同反应条件对目标化合物 51 合成产率的影响

Table 1 The effects of reaction conditions on the synthesis of the title compound $\mathbf{5 l}$

\begin{tabular}{ccccr}
\hline Entry & Base & Solvent & Reaction condition & Yield/\% \\
\hline 1 & Pyridine & $\mathrm{CH}_{3} \mathrm{COCH}_{3}$ & Reflux & 0 \\
2 & $\mathrm{Na}_{2} \mathrm{CO}_{3}$ & $\mathrm{CH}_{3} \mathrm{COCH}_{3}$ & Reflux & 15 \\
3 & $\mathrm{Na}_{2} \mathrm{CO}_{3}$ & $\mathrm{CH}_{3} \mathrm{CN}$ & Reflux & 27 \\
4 & $\mathrm{~K}_{2} \mathrm{CO}_{3}$ & $\mathrm{CH}_{3} \mathrm{CN}$ & Reflux & 50 \\
5 & $\mathrm{~K}_{2} \mathrm{CO}_{3}$ & DMF & $t=80{ }^{\circ} \mathrm{C}$ & 45 \\
\hline
\end{tabular}
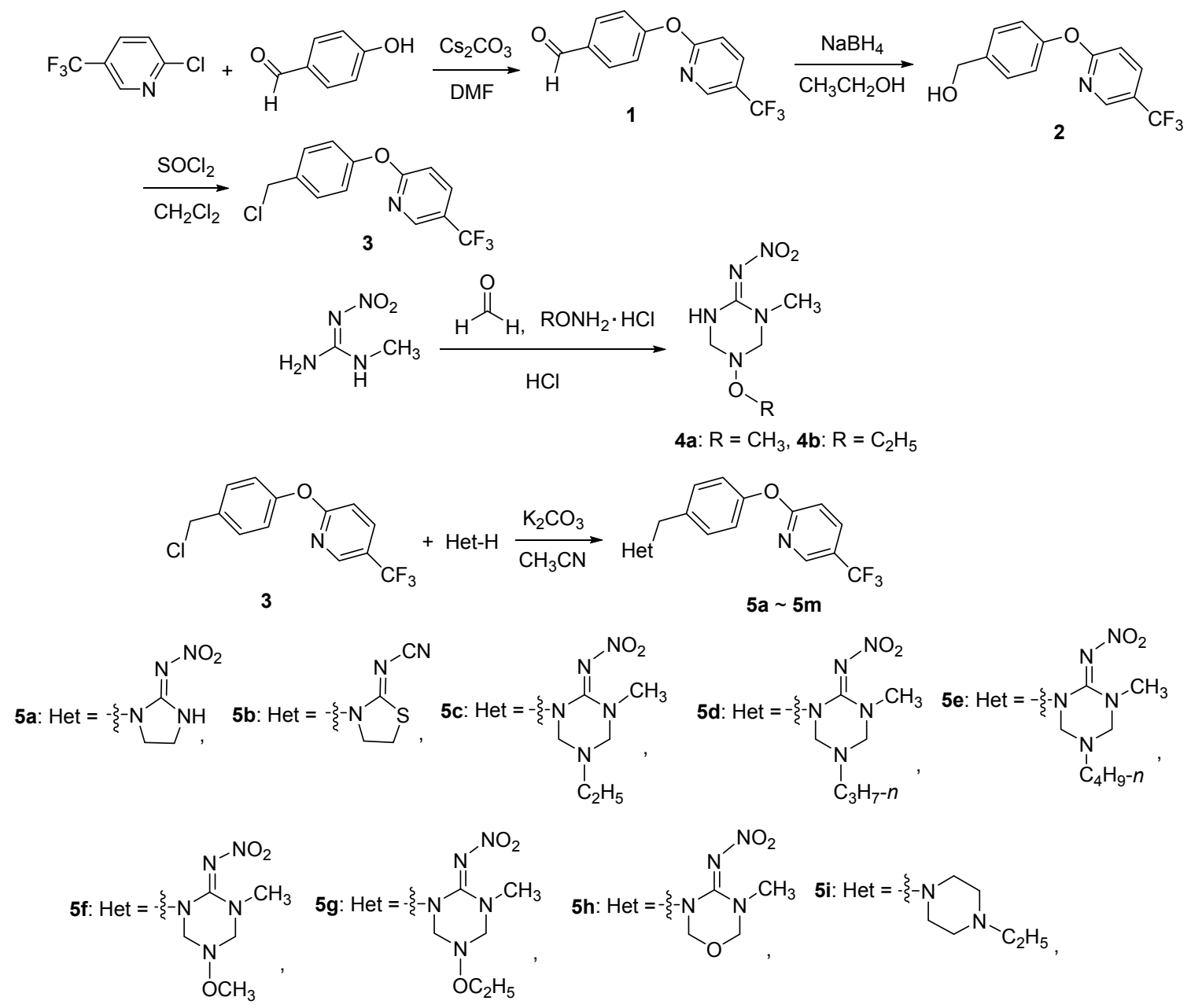<smiles>[SiH3]C=CN1CCN(c2ccccc2)CC1</smiles>

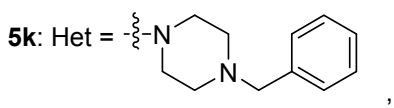<smiles>[Hg]=[Al]N1CCOCC1</smiles><smiles>[SnH]=C[AsH2]N1CCCCC1</smiles>

图式 1 目标化合物 5 的合成路线

Scheme 1 The synthetic route of title compounds 5 


\section{2 目标化合物的图谱剖析}

分别以目标化合物 5a、5c 的核磁氢谱与碳谱数据 为例进行解析. 对于化合物 $\mathbf{5 a}, \delta$ 在 8.44 处的单峰为吡 啶环上一个氢的吸收峰; $\delta 8.19$ 处的单峰对应于咪唑烷 环上 $\mathrm{NH}$ 的氢的吸收峰; $\delta 7.94$ 处的双重峰为吡啶环上一 个氢的吸收峰; $\delta 7.38$ 处的双重峰为苯环上两个氢的吸 收峰; $\delta 7.16$ 处的双重峰为苯环上两个氢的吸收峰; $\delta$ 7.05 处的双重峰为吡啶环上一个氢的吸收峰; $\delta 4.58$ 处 的单峰为与苯基相连的 $\mathrm{CH}_{2}$ 上两个氢的吸收峰; $\delta 3.81$ 处的三重峰为咪唑烷环上一个 $\mathrm{CH}_{2}$ 两个氢的吸收峰; $\delta$ 3.57 处的三重峰对应于咪唑烷环上另外一个 $\mathrm{CH}_{2}$ 两个氢 的吸收峰; $\delta$ 在 45.2 处的峰为与苯环相连的 $\mathrm{CH}_{2}$ 碳原子 的信号峰; $\delta$ 在 48.0 和 41.4 处的峰分别对应于咪唑烷环 上两个 $\mathrm{CH}_{2}$ 碳原子的信号峰. 对于化合物 $\mathbf{5 c}, \delta$ 在 8.33 处的单峰为吡啶环上一个氢的吸收峰; $\delta 7.86$ 处的双重 峰为吡啶环上一个氢的吸收峰; $\delta 7.37$ 处的双重峰为苯 环上两个氢的吸收峰; $\delta 7.09$ 处的双重峰为苯环上两个 氢的吸收峰; $\delta 6.97$ 处的双重峰为吡啶环上一个氢的吸 收峰; $\delta 4.62$ 处的单峰为与苯基相连的 $\mathrm{CH}_{2}$ 上两个氢的 吸收峰; $\delta 4.34$ 处的单峰为三嗪环上一个 $\mathrm{CH}_{2}$ 两个氢的 吸收峰; $\delta 4.26$ 处的单峰对应于三嗪环上另外一个 $\mathrm{CH}_{2}$ 两个氢的吸收峰; $\delta 3.02$ 处的单峰对应于三嗪环 $\mathrm{NCH}_{3}$ 上 三个氢的吸收峰; $\delta 2.62$ 处的四重峰对应于三嗪环 $\mathrm{NCH}_{2} \mathrm{CH}_{3}$ 上 $\mathrm{CH}_{2}$ 两个氢的吸收峰; $\delta 0.86$ 处的三重峰对 应于三嗪环 $\mathrm{NCH}_{2} \mathrm{CH}_{3}$ 上 $\mathrm{CH}_{3}$ 三个氢的吸收峰; $\delta$ 在 68.6 和 64.0 处的峰分别对应于三嗪环上两个 $\mathrm{CH}_{2}$ 碳原子的 信号峰; $\delta$ 在 51.1 处的峰为与苯环相连的 $\mathrm{CH}_{2}$ 碳原子的
信号峰; $\delta 45.5$ 处的峰对应于三嗪环 $\mathrm{NCH}_{2} \mathrm{CH}_{3}$ 上 $\mathrm{CH}_{2}$ 碳 原子的信号峰; $\delta 35.3$ 处的峰对应于三嗪环 $\mathrm{NCH}_{3}$ 上 $\mathrm{CH}_{3}$ 碳原子的信号峰; $\delta 12.8$ 处的峰对应于三嗪环 $\mathrm{NCH}_{2} \mathrm{CH}_{3}$ 上 $\mathrm{CH}_{3}$ 碳原子的信号峰.

\section{3 化合物的杀虫活性}

目标化合物 $5 \mathrm{a} \sim \mathbf{5 m}$ 对小菜蛾(Plutella xylostella)、 蚜虫 (Aphis craccivora)、朱砂叶螨 (Tetranychus cinnabarinus) 和褐飞闽(Nilaparvata lugens)的生物活性结果 如表 2 所示. 初步的生测结果表明, 大多数目标化合物 对小菜蛾表现出一定的杀虫活性，但并无明显的规律. 在五元杂环中，当含氮杂环为 2 -氰基亚胺基-1,3-噻唑烷 的化合物 $\mathbf{5 b}$ 在测试浓度为 $200 \mu \mathrm{g} / \mathrm{mL}$ 时对小菜蛾的杀 死率为 $100 \%$; 当含氮杂环为三嗪环时, 在 5 -烷基取代 物中, 5-正丙基取代物 $\mathbf{5 d}$ 在测试浓度为 $200 \mu \mathrm{g} / \mathrm{mL}$ 时对 小菜蛾的防治效果(100\%)要优于 5-乙基取代物 5c(85\%) 和 5-正丁基取代物 5e (63\%); 在 5-烷氧基取代物中，化 合物 $5 \mathbf{f}$ 和 $5 \mathrm{~g}$ 在测试浓度为 $200 \mu \mathrm{g} / \mathrm{mL}$ 时对小菜蛾的杀 死率均达 $100 \%$; 当含氮杂环为取代哌嗪环时, $N$-芐基取 代物 5k 在测试浓度为 $200 \mu \mathrm{g} / \mathrm{mL}$ 时对小菜蛾的杀灭效 果(100\%)要优于 $N$-乙基取代物 $\mathbf{5 i}(67 \%)$ 和 $N$-苯基取代物 $5 \mathbf{j}(60 \%)$. 整体来看, 当含氮杂环部分为取代三嗪环的 目标物对小菜蛾的防治效果要略高于含其它杂环的目 标物. 另外，部分标题化合物对蚜虫也显示出一定的杀 虫作用，其中化合物 $\mathbf{5 c}$ 和 $\mathbf{5 f}$ 在测试浓度为 $200 \mu \mathrm{g} / \mathrm{mL}$ 时对蚜虫的杀死率为 $45 \%$ 和 $85 \%$. 因此，该结构类型化 合物可作进一步的结构优化，以寻找与发现生物活性更 好的化合物.

表 2 目标化合物 $\mathbf{5 a} \sim 5 \mathrm{~m}$ 的杀虫与杀螨活性 ${ }^{a}$

Table 2 Insecticidal and acarcidal activities of target compounds $\mathbf{5 a} \sim \mathbf{5} \mathbf{m}$ at $200 \mu \mathrm{g} / \mathrm{mL}$

\begin{tabular}{|c|c|c|c|c|}
\hline \multirow{2}{*}{ Compd. } & \multicolumn{4}{|c|}{ Mortality $/ \%$} \\
\hline & Plutella xylostella & Aphis craccivora & Tetranychus cinnabarinus & Nilaparvata lugens \\
\hline $5 \mathbf{5 a}$ & 0 & 0 & 0 & 0 \\
\hline $5 \mathbf{b}$ & 100 & 24 & 0 & 0 \\
\hline $5 c$ & 85 & 45 & 0 & 0 \\
\hline $5 d$ & 100 & 25 & 0 & 0 \\
\hline $5 e$ & 63 & 18 & 21 & 0 \\
\hline $5 f$ & 100 & 85 & 0 & 0 \\
\hline $5 g$ & 100 & 20 & 0 & 0 \\
\hline $5 \mathbf{h}$ & 0 & 0 & 0 & 0 \\
\hline $5 \mathbf{i}$ & 67 & 27 & 0 & 23 \\
\hline $\mathbf{5 j}$ & 60 & 22 & 0 & 0 \\
\hline $5 \mathbf{k}$ & 100 & 30 & 0 & 0 \\
\hline 51 & 0 & 0 & 0 & 0 \\
\hline $5 \mathrm{~m}$ & 0 & 0 & 0 & 0 \\
\hline Pyridalyl & 100 & - & - & - \\
\hline Imidacloprid & - & 100 & - & 100 \\
\hline Fenpyroximate & - & - & 100 & - \\
\hline
\end{tabular}

a“_." refers to "not tested". 


\section{4 化合物的抗肿瘤活性}

目标化合物 $5 \mathrm{a} \sim \mathbf{5 m}$ 对人胰腺癌(Panc-1)、人肝癌 (HUH-7)、人结肠癌(HCT-116)和人乳腺癌 $(\mathrm{MCF}-7)$ 细胞 株的体外抗肿瘤活性结果如表 3 所示. 测试结果显示, 部分标题化合物对四种癌细胞株具有一定的抑制效果. 在五元杂环中, 当含氮杂环为 2-氭基亚胺基-1,3-噻唑烷 的化合物 $\mathbf{5 b}$ 对四种癌细胞株的抑制活性相对较高, 尤 其是对 $\mathrm{HUH}-7$ 和 MCF-7 细胞株, 其 $\mathrm{IC}_{50}$ 值分别为 12.98 和 $18.45 \mu \mathrm{mol} / \mathrm{L}$, 分别与阳性对照药顺铂(Cisplatin)和 5氟尿嘧啶(5-Fluorouracil)的活性相近; 当含氮杂环为三 嗪环时，在 5-烷基取代物中, 5-乙基取代物 5c 对 Panc-1、 HUH-7 和 MCF-7 细胞株的抑制活性相对较高; 在 5-烷 氧基取代物中，5-甲氧基取代物 5f 对 Panc-1、HUH-7、 HCT-116 和 MCF-7 细胞株的抗肿瘤活性要明显优于 5乙氧基取代物 $\mathbf{5 g}$, 其 $\mathrm{IC}_{50}$ 值分别为 $8.29,10.64,23.48$ 和 $18.31 \mu \mathrm{mol} / \mathrm{L}$, 分别与阳性对照药索拉菲尼(Sorafenib), 顺铂(Cisplatin)和 5-氟尿嘧啶(5-Fluorouracil)的活性相 当; 当含氮杂环为取代基哌嗪环时, $N$-茮基取代物 $\mathbf{5 k}$ 对 HUH-7、HCT-116 和 MCF-7 细胞株抑制活性要优于 $N-$ 乙基取代物 $\mathbf{5 i}$ 和 $N$-苯基取代物 $\mathbf{5 j}$, 尤其对 HCT-116 细 胞株的抑制值 $\mathrm{IC}_{50}$ 为 $13.20 \mu \mathrm{mol} / \mathrm{L}$, 要高于阳性对照药 5-氟尿嘧啶(5-Fluorouracil)的活性. 总体来看, 化合物 $\mathbf{5 f}$ 和 $5 \mathrm{k}$ 对四种癌细胞株的抑制活性相对较高, 具备进一 步研究的潜力.

表 3 目标化合物 $\mathbf{5 a} \sim \mathbf{5 m}$ 的体外抗肿瘤活性 ${ }^{a}$

Table 3 The cytotoxicity of target compounds $\mathbf{5 a} \sim \mathbf{5 m}$

\begin{tabular}{lcccc}
\hline \multirow{2}{*}{ Compd. } & \multicolumn{4}{c}{$\mathrm{IC}_{50} / \mu \mathrm{mol} / \mathrm{L}$} \\
\cline { 2 - 5 } & Panc-1 & HUH-7 & $\mathrm{HCT}-116$ & $\mathrm{MCF}-7$ \\
\hline $\mathbf{5 a}$ & $>50$ & $>50$ & $>50$ & $>50$ \\
$\mathbf{5 b}$ & 15.85 & 12.98 & 36.22 & 18.45 \\
$\mathbf{5 c}$ & 13.95 & 15.25 & 36.57 & 29.14 \\
$\mathbf{5 d}$ & $>50$ & 26.40 & 37.12 & $>50$ \\
$\mathbf{5 e}$ & $>50$ & 29.32 & 33.19 & $>50$ \\
$\mathbf{5 f}$ & 8.29 & 10.64 & 23.48 & 18.31 \\
$\mathbf{5 g}$ & $>50$ & 40.62 & 36.51 & $>50$ \\
$\mathbf{5 h}$ & $>50$ & $>50$ & $>50$ & $>50$ \\
$\mathbf{5 i}$ & 12.15 & 33.89 & 18.42 & 21.06 \\
$\mathbf{5 j}$ & 27.15 & 23.09 & 35.03 & 30.21 \\
$\mathbf{5 k}$ & 11.91 & 16.27 & 13.20 & 15.31 \\
$\mathbf{5 l}$ & $>50$ & $>50$ & $>50$ & $>50$ \\
$\mathbf{5 m}$ & $>50$ & $>50$ & $>50$ & $>50$ \\
5-Fluorouracil & - & - & 29.50 & 18.39 \\
Cisplatin & - & 12.70 & - & - \\
Sorafenib & 11.50 & - & - & - \\
\hline a“-."refers to "not tested”. & & &
\end{tabular}

\section{2 结论}

本文运用活性亚结构拼接原理, 设计并制备了一系 列新型含氮杂环结构的吡啶苯醚类衍生物. 初步的生物
活性测试结果显示, 部分目标物对小菜蛾和蚜虫表现出 较好的杀虫活性，其中化合物 5b、5c、5d、5f、5 g 和 5k 在测试浓度为 $200 \mu \mathrm{g} / \mathrm{mL}$ 时对小菜蛾的防治效果分 别为 $100 \%, 85 \%, 100 \%, 100 \%, 100 \%$ 和 $100 \%$; 化合物 $\mathbf{5 f}$ 在测试浓度为 $200 \mu \mathrm{g} / \mathrm{mL}$ 时对蚜虫的杀死率为 $85 \%$. 此 外, 部分标题化合物对人肤腺癌、人肝癌、人结肠癌和 人乳腺癌细胞株具有明显的抗肿瘤活性，其中化合物 $\mathbf{5 f}$ 和 $5 \mathrm{k}$ 对人肤腺癌、人肝癌、人结肠癌和人乳腺癌细胞 株的 $\mathrm{IC}_{50}$ 值分别与阳性对照药索拉菲尼(Sorafenib), 顺 铂(Cisplatin)或 5-氟尿嘧啶 (5-Fluorouracil)的数值相近, 具有进一步研究的价值. 这为今后进一步从事吡啶苯醚 类化合物的结构优化与生物活性研究提供了重要的实 验数据.

\section{3 实验部分}

\section{1 仪器与试剂}

X-4 型数字显示熔点测定仪(北京泰克仪器有限公 司), 温度计未经校正; Yanaco-CHN CORDER MT-3 自 动元素分析仪; Bruker AM-400 型核磁共振仪, 以 $\mathrm{CDCl}_{3}$ 为溶剂, TMS 为内标; Shimadzu-435 型红外光谱仪 $(\mathrm{KBr}$ 压片); 柱层析硅胶为 $\mathrm{H}$ 型(青岛海洋化工厂，200～300 目). 所用试剂均为分析纯. 中间体 4-(5-三氟甲基吡啶2-基氧基)苯甲醛(1)按文献[25]方法制备，4-(5-三氟甲基 吡啶-2-基氧基)苯甲醇(2)和 4-(5-三氟甲基吡啶-2-基氧 基)苯甲基氯(3)参照文献[26, 27]方法合成.

\section{2 中间体 5-烷氧基-3-甲基- $N$-硝基-1H-1,3,5-三嗪-2 亚胺(4)的合成}

以化合物 $4 \mathrm{a}$ 的合成为例: 在一 $250 \mathrm{~mL}$ 反应瓶中, 加入 $20 \mathrm{mmol}$ 中间体 $N$-甲基- $N$ - 硝基胍、160 $\mathrm{mmol} 37 \%$ 甲醛溶液及 $60 \mathrm{~mL}$ 乙醇, 摚拌下向其中依次加入 40 $\mathrm{mmol}$ 甲氧基胺盐酸盐、 $80 \mathrm{mmol}$ 浓盐酸, 加毕, 将反应 混合物缓慢升温至回流. TLC 跟踪, 待原料点消失后停 止加热. 静置, 析出大量固体, 抽滤, 所得固体用乙酸 乙酯重结晶得白色固体, 产率 $40 \%{ }^{[28]}$. m.p. $158 \sim 160{ }^{\circ} \mathrm{C}$; ${ }^{1} \mathrm{H}$ NMR (400 MHz, $\mathrm{CDCl}_{3}$ ) $\delta: 3.08$ (s, $\left.3 \mathrm{H}, \mathrm{NCH}_{3}\right), 3.65$ (s, $\left.3 \mathrm{H}, \mathrm{OCH}_{3}\right), 4.35 \sim 4.60\left(\mathrm{~m}, 4 \mathrm{H}, 2 \times \mathrm{CH}_{2}\right), 9.61(\mathrm{~s}, 1 \mathrm{H}$, $\mathrm{NH})$. 采用类似的方法合成了中间体 $\mathbf{4 b}$.

\section{3 目标化合物 5 的合成}

在一 $50 \mathrm{~mL}$ 圆底烧瓶中，加入 $2 \mathrm{mmol}$ 中间体 3、2.2 $\mathrm{mmol}$ 含氮杂环、 $4 \mathrm{mmol}$ 无水碳酸钾及 $25 \mathrm{~mL}$ 乙腈. 加 毕，将反应混合物缓慢升温至回流. TLC 监测反应完毕. 待反应液冷却至室温，抽滤，蒸除溶剂，所得残余物经 柱层析 $[V$ (石油醚) $: V$ (乙酸乙酯 $)=8: 1]$ 分离, 得到目 标化合物 $\mathbf{5 a} \sim 5 \mathbf{m}$. 
1-[4-(5-三氟甲基吡啶-2-基氧基)苯甲基]- $N$-硝基咪 唑烷-2-亚胺 (5a): 白色固体, 产率 66\%. m.p. 160 $162^{\circ} \mathrm{C} ;{ }^{1} \mathrm{H}$ NMR $\left(400 \mathrm{MHz}, \mathrm{CDCl}_{3}\right) \delta: 3.57(\mathrm{t}, J=8.8 \mathrm{~Hz}$, $\left.2 \mathrm{H}, \mathrm{CH}_{2}\right), 3.81\left(\mathrm{t}, J=8.8 \mathrm{~Hz}, 2 \mathrm{H}, \mathrm{CH}_{2}\right), 4.58\left(\mathrm{~s}, 2 \mathrm{H}, \mathrm{CH}_{2}\right)$, $7.05(\mathrm{~d}, J=8.4 \mathrm{~Hz}, 1 \mathrm{H}, \mathrm{Py}-\mathrm{H}), 7.16(\mathrm{~d}, J=7.2 \mathrm{~Hz}, 2 \mathrm{H}$, Ar-H), 7.38 (d, $J=7.6 \mathrm{~Hz}, 2 \mathrm{H}, \mathrm{Ar}-\mathrm{H}), 7.94$ (d, $J=8.4 \mathrm{~Hz}$, $1 \mathrm{H}, \mathrm{Py}-\mathrm{H}), 8.19$ (s, 1H, NH), 8.44 (s, 1H, Py-H); ${ }^{13} \mathrm{C}$ NMR $\left(100 \mathrm{MHz}, \mathrm{CDCl}_{3}\right) \delta: 41.4,45.2,48.0,111.6,121.9,129.9$, $132.0,136.8,136.9,145.3,145.4,153.1,161.3,165.5$. Anal. calcd for $\mathrm{C}_{16} \mathrm{H}_{14} \mathrm{~F}_{3} \mathrm{~N}_{5} \mathrm{O}_{3}: \mathrm{C}$ 50.40, H 3.70, N 18.37; found C 50.58, H 3.56, N 18.21.

1-[4-(5-三氟甲基吡啶-2-基氧基)苯甲基]- $N$-氭基噻 唑烷-2-亚胺 (5b): 白色固体, 产率 62\%. m.p. 175 $176^{\circ} \mathrm{C} ;{ }^{1} \mathrm{H}$ NMR $\left(400 \mathrm{MHz}, \mathrm{CDCl}_{3}\right) \delta: 3.37(\mathrm{t}, J=7.6 \mathrm{~Hz}$, $\left.2 \mathrm{H}, \mathrm{CH}_{2}\right), 3.82\left(\mathrm{t}, J=7.6 \mathrm{~Hz}, 2 \mathrm{H}, \mathrm{CH}_{2}\right), 4.61\left(\mathrm{~s}, 2 \mathrm{H}, \mathrm{CH}_{2}\right)$, $7.02(\mathrm{~d}, J=8.4 \mathrm{~Hz}, 1 \mathrm{H}, \mathrm{Py}-\mathrm{H}), 7.13(\mathrm{~d}, J=7.2 \mathrm{~Hz}, 2 \mathrm{H}$, Ar-H), 7.32 (d, $J=8.0 \mathrm{~Hz}, 2 \mathrm{H}, \mathrm{Ar}-\mathrm{H}), 7.89$ (d, $J=8.8 \mathrm{~Hz}$, 1H, Py-H), 8.39 (s, 1H, Py-H); ${ }^{13} \mathrm{C}$ NMR (100 MHz, $\left.\mathrm{CDCl}_{3}\right) \delta: 27.2,49.8,52.3,111.7,117.5,122.0,125.0$, 129.9, 136.8, 136.9, 145.2, 145.3, 153.2, 165.4, 174.5. Anal. calcd for $\mathrm{C}_{17} \mathrm{H}_{13} \mathrm{~F}_{3} \mathrm{~N}_{4} \mathrm{OS}$ : C 53.96, H 3.46, N 14.81; found C 53.78, H 3.62, N 14.95 .

1-[4-(5-三氟甲基吡啶-2-基氧基)苯甲基]-5-乙基-3甲基- $N$ - 硝基-1,3,5-三嗪-2-亚胺 $(\mathbf{5 c})$ ：白色固体, 产率 $56 \%$. m.p. $156 \sim 158{ }^{\circ} \mathrm{C} ;{ }^{1} \mathrm{H}$ NMR $\left(400 \mathrm{MHz}, \mathrm{CDCl}_{3}\right) \delta$ : $0.86\left(\mathrm{t}, J=6.0 \mathrm{~Hz}, 3 \mathrm{H}, \mathrm{CH}_{3}\right), 2.62(\mathrm{q}, J=6.0 \mathrm{~Hz}, 2 \mathrm{H}$, $\left.\mathrm{CH}_{2}\right), 3.02\left(\mathrm{~s}, 3 \mathrm{H}, \mathrm{N}-\mathrm{CH}_{3}\right), 4.26$ (s, $\left.2 \mathrm{H}, \mathrm{CH}_{2}\right), 4.34$ (s, $2 \mathrm{H}$, $\left.\mathrm{CH}_{2}\right), 4.62\left(\mathrm{~s}, 2 \mathrm{H}, \mathrm{CH}_{2}\right), 6.97(\mathrm{~d}, J=8.0 \mathrm{~Hz}, 1 \mathrm{H}, \mathrm{Py}-\mathrm{H})$, 7.09 (d, $J=8.0 \mathrm{~Hz}, 2 \mathrm{H}, \mathrm{Ar}-\mathrm{H}), 7.37(\mathrm{~d}, J=8.0 \mathrm{~Hz}, 2 \mathrm{H}$, Ar-H), 7.86 (d, $J=8.0 \mathrm{~Hz}, 1 \mathrm{H}, \mathrm{Py}-\mathrm{H}), 8.33$ (s, $1 \mathrm{H}, \mathrm{Py}-\mathrm{H})$; ${ }^{13} \mathrm{C}$ NMR $\left(100 \mathrm{MHz}, \mathrm{CDCl}_{3}\right) \delta: 12.8,35.3,45.5,51.1$, 64.0, 68.6, 111.5, 122.0, 125.0, 130.2, 131.6, 136.7, 136.8, 145.2, 153.2, 157.3, 165.4. Anal. calcd for $\mathrm{C}_{19} \mathrm{H}_{21} \mathrm{~F}_{3} \mathrm{~N}_{6} \mathrm{O}_{3}$ : C 52.05, H 4.83, N 19.17; found C 52.21, H 4.72, N 19.03.

1-[4-(5-三氟甲基吡啶-2-基氧基)苯甲基]-5-正丙基3-甲基- $N$-硝基-1,3,5-三嗪-2-亚胺(5d): 白色固体, 产率 $54 \%$. m.p. $160 \sim 162{ }^{\circ} \mathrm{C} ;{ }^{1} \mathrm{H}$ NMR $\left(400 \mathrm{MHz}, \mathrm{CDCl}_{3}\right) \delta$ : $0.76\left(\mathrm{t}, J=7.2 \mathrm{~Hz}, 3 \mathrm{H}, \mathrm{CH}_{3}\right), 1.17 \sim 1.26\left(\mathrm{~m}, 2 \mathrm{H}, \mathrm{CH}_{2}\right)$, 2.49 (t, $\left.J=7.2 \mathrm{~Hz}, 2 \mathrm{H}, \mathrm{CH}_{2}\right), 3.02\left(\mathrm{~s}, 3 \mathrm{H}, \mathrm{NCH}_{3}\right), 4.21$ (s, $\left.2 \mathrm{H}, \mathrm{CH}_{2}\right), 4.29$ (s, $2 \mathrm{H}, \mathrm{CH}_{2}$ ), 4.61 (s, $\left.2 \mathrm{H}, \mathrm{CH}_{2}\right), 6.96$ (d, $J=8.4 \mathrm{~Hz}, 1 \mathrm{H}, \mathrm{Py}-\mathrm{H}$ ), 7.08 (d, $J=8.4 \mathrm{~Hz}, 2 \mathrm{H}, \mathrm{Ar}-\mathrm{H}$ ), 7.33 (d, $J=8.4 \mathrm{~Hz}, 2 \mathrm{H}, \mathrm{Ar}-\mathrm{H}), 7.85(\mathrm{~d}, J=8.0 \mathrm{~Hz}, 1 \mathrm{H}, \mathrm{Py}-\mathrm{H})$, 8.33 (s, $1 \mathrm{H}, \mathrm{Py}-\mathrm{H}) ;{ }^{13} \mathrm{C}$ NMR $\left(100 \mathrm{MHz}, \mathrm{CDCl}_{3}\right) \delta: 6.0$, 15.6, 30.2, 46.1, 48.0, 59.2, 63.9, 106.3, 116.8, 125.0, 126.3, 131.5, 131.6, 140.0, 140.1, 148.1, 152.3, 160.2; IR
(KBr) v: 3070, 3047, 2965, 2937, 2877, 1604, 1511, 1489, 1290, 1210, 1164, 1081, 1013, 959, 857, 777, 757, 582 $\mathrm{cm}^{-1}$. Anal. calcd for $\mathrm{C}_{20} \mathrm{H}_{23} \mathrm{~F}_{3} \mathrm{~N}_{6} \mathrm{O}_{3}: \mathrm{C} 53.09, \mathrm{H} 5.12, \mathrm{~N}$ 18.58; found C 53.28, H 5.01, N 18.42.

1-[4-(5-三氟甲基吡啶-2-基氧基)苯甲基]-5-正丁基3-甲基- $N$-硝基-1,3,5-三嗪-2-亚胺(5e)：白色固体, 产率 50\%. m.p. $154 \sim 156{ }^{\circ} \mathrm{C} ;{ }^{1} \mathrm{H}$ NMR $\left(400 \mathrm{MHz}, \mathrm{CDCl}_{3}\right) \delta$ : $0.76\left(\mathrm{t}, J=7.2 \mathrm{~Hz}, 3 \mathrm{H}, \mathrm{CH}_{3}\right), 1.14 \sim 1.17\left(\mathrm{~m}, 4 \mathrm{H}, 2 \times \mathrm{CH}_{2}\right)$, $2.51\left(\mathrm{t}, J=6.8 \mathrm{~Hz}, 2 \mathrm{H}, \mathrm{CH}_{2}\right), 3.00\left(\mathrm{~s}, 3 \mathrm{H}, \mathrm{NCH}_{3}\right), 4.20(\mathrm{~s}$, $\left.2 \mathrm{H}, \mathrm{CH}_{2}\right), 4.28\left(\mathrm{~s}, 2 \mathrm{H}, \mathrm{CH}_{2}\right), 4.59\left(\mathrm{~s}, 2 \mathrm{H}, \mathrm{CH}_{2}\right), 6.94(\mathrm{~d}$, $J=8.8 \mathrm{~Hz}, 1 \mathrm{H}, \mathrm{Py}-\mathrm{H}$ ), 7.07 (d, $J=8.4 \mathrm{~Hz}, 2 \mathrm{H}, \mathrm{Ar}-\mathrm{H}), 7.33$ (d, $J=8.8 \mathrm{~Hz}, 2 \mathrm{H}, \operatorname{Ar}-\mathrm{H}), 7.84(\mathrm{~d}, J=8.8 \mathrm{~Hz}, 1 \mathrm{H}, \mathrm{Py}-\mathrm{H})$, 8.32 (s, $1 \mathrm{H}, \mathrm{Py}-\mathrm{H}) ;{ }^{13} \mathrm{C}$ NMR $\left(100 \mathrm{MHz}, \mathrm{CDCl}_{3}\right) \delta: 8.4$, 14.6, 24.4, 30.2, 45.8, 46.0, 59.2, 63.9, 106.3, 116.7, 125.0, $126.3,131.5,131.6,140.0,140.1,148.1,152.2,160.2$. Anal. calcd for $\mathrm{C}_{21} \mathrm{H}_{25} \mathrm{~F}_{3} \mathrm{~N}_{6} \mathrm{O}_{3}$ : C 54.07, H 5.40, N 18.02; found C 54.21, H 5.22, N 17.83 .

1-[4-(5-三氟甲基吡啶-2-基氧基)苯甲基]-5-甲氧基3-甲基- $N$-硝基-1,3,5-三嗪-2-亚胺(5f)：白色固体, 产率 $48 \%$. m.p. $152 \sim 154{ }^{\circ} \mathrm{C} ;{ }^{1} \mathrm{H}$ NMR $\left(400 \mathrm{MHz}, \mathrm{CDCl}_{3}\right) \delta$ : $3.12\left(\mathrm{~s}, 3 \mathrm{H}, \mathrm{NCH}_{3}\right), 3.49\left(\mathrm{~s}, 3 \mathrm{H}, \mathrm{OCH}_{3}\right), 4.41 \sim 4.92(\mathrm{~m}$, $\left.6 \mathrm{H}, 3 \times \mathrm{CH}_{2}\right), 7.02(\mathrm{~d}, J=8.8 \mathrm{~Hz}, 1 \mathrm{H}, \mathrm{Py}-\mathrm{H}), 7.14(\mathrm{~d}, J=$ $8.4 \mathrm{~Hz}, 2 \mathrm{H}, \mathrm{Ar}-\mathrm{H}), 7.40$ (d, J=8.4 Hz, 2H, Ar-H), 7.92 (d, $J=8.8 \mathrm{~Hz}, 1 \mathrm{H}, \mathrm{Py}-\mathrm{H}), 8.41$ (s, $1 \mathrm{H}, \mathrm{Py}-\mathrm{H}) ;{ }^{13} \mathrm{C}$ NMR $(100$ $\left.\mathrm{MHz}, \mathrm{CDCl}_{3}\right) \delta: 36.3,51.9,60.2,65.3,69.3,111.5,121.9$, $124.7,129.9,131.3,136.8,145.3,145.4,153.2,157.6$, 165.5. Anal. calcd for $\mathrm{C}_{18} \mathrm{H}_{19} \mathrm{~F}_{3} \mathrm{~N}_{6} \mathrm{O}_{4}$ : C 49.09, H 4.35, N 19.08; found C 48.92, H 4.54, N 19.26.

1-[4-(5-三氟甲基吡啶-2-基氧基)苯甲基]-5-乙氧基3-甲基- $N$-硝基-1,3,5-三嗪-2-亚胺 (5g)：白色固体，产率 $52 \%$. m.p. $127 \sim 129{ }^{\circ} \mathrm{C} ;{ }^{1} \mathrm{H}$ NMR $\left(400 \mathrm{MHz}, \mathrm{CDCl}_{3}\right) \delta$ : $1.10\left(\mathrm{t}, J=6.8 \mathrm{~Hz}, 3 \mathrm{H}, \mathrm{CH}_{3}\right), 3.12\left(\mathrm{~s}, 3 \mathrm{H}, \mathrm{NCH}_{3}\right), 3.71(\mathrm{q}$, $\left.J=7.2 \mathrm{~Hz}, 2 \mathrm{H}, \mathrm{OCH}_{2}\right), 4.35 \sim 4.98\left(\mathrm{~m}, 6 \mathrm{H}, 3 \times \mathrm{CH}_{2}\right), 7.05$ (d, $J=8.0 \mathrm{~Hz}, 1 \mathrm{H}, \mathrm{Py}-\mathrm{H}), 7.13$ (d, $J=8.0 \mathrm{~Hz}, 2 \mathrm{H}, \mathrm{Ar}-\mathrm{H})$, $7.44(\mathrm{~d}, J=8.0 \mathrm{~Hz}, 2 \mathrm{H}, \mathrm{Ar}-\mathrm{H}), 7.94(\mathrm{~d}, J=8.8 \mathrm{~Hz}, 1 \mathrm{H}$, Py-H), 8.40 (s, $1 \mathrm{H}, \mathrm{Py}-\mathrm{H}) ;{ }^{13} \mathrm{C}$ NMR (100 MHz, $\left.\mathrm{CDCl}_{3}\right) \delta$ : 13.7, 36.0, 51.4, 66.0, 68.1, 69.9, 111.5, 121.7, 125.0, $129.6,129.7,131.4,136.7,136.8,145.1,145.2,152.9$, 157.3, 165.5; IR (KBr) v: 3074, 3051, 2982, 2928, 2870, 1600, 1515, 1492, 1263, 1202, 1161, 1078, 1046, 891, 830, $764,530 \mathrm{~cm}^{-1}$. Anal. calcd for $\mathrm{C}_{19} \mathrm{H}_{21} \mathrm{~F}_{3} \mathrm{~N}_{6} \mathrm{O}_{4}: \mathrm{C} 50.22, \mathrm{H}$ 4.66, N 18.49; found C 50.37, H 4.48, N 18.35.

1-[4-(5-三氟甲基吡啶-2-基氧基)苯甲基]-5-甲基- $N$ 硝基-1,3,5-噁二嗪-4-亚胺(5h): 白色固体，产率 64\%. m.p. $138 \sim 140{ }^{\circ} \mathrm{C} ;{ }^{1} \mathrm{H}$ NMR (400 MHz, $\mathrm{CDCl}_{3}$ ) $\delta: 3.07$ (s, 
$\left.3 \mathrm{H}, \mathrm{NCH}_{3}\right), 4.72\left(\mathrm{~s}, 2 \mathrm{H}, \mathrm{CH}_{2}\right), 4.86\left(\mathrm{~s}, 2 \mathrm{H}, \mathrm{CH}_{2}\right), 4.92$ (s, 2H, $\mathrm{CH}_{2}$ ), 7.02 (d, J=8.4 Hz, 1H, Py-H), 7.14 (d, $J=7.2$ $\mathrm{Hz}, 2 \mathrm{H}, \mathrm{Ar}-\mathrm{H}), 7.39$ (d, J=7.6 Hz, 2H, Ar-H), 7.91 (d, $J=$ $8.8 \mathrm{~Hz}, 1 \mathrm{H}, \mathrm{Py}-\mathrm{H}), 8.41$ (s, 1H, Py-H); ${ }^{13} \mathrm{C}$ NMR $(100$ $\left.\mathrm{MHz}, \mathrm{CDCl}_{3}\right) \delta: 34.5,51.3,77.1,79.8,111.6,122.0,129.6$, 131.3, 136.8, 136.9, 145.3, 145.4, 153.3, 157.8, 165.4 . Anal. calcd for $\mathrm{C}_{17} \mathrm{H}_{16} \mathrm{~F}_{3} \mathrm{~N}_{5} \mathrm{O}_{4}$ : C 49.64, H 3.92, N 17.03; found $\mathrm{C} 49.82, \mathrm{H} 3.73, \mathrm{~N} 17.21$.

1-[4-(5-三氟甲基吡啶-2-基氧基)苯甲基]-4-乙基哌 嗪(5i): 白色固体, 产率 55\%. m.p. 78 80 ${ }^{\circ} \mathrm{C} ;{ }^{1} \mathrm{H}$ NMR $\left(400 \mathrm{MHz}, \mathrm{CDCl}_{3}\right) \delta: 1.10\left(\mathrm{t}, J=8.0 \mathrm{~Hz}, 3 \mathrm{H}, \mathrm{CH}_{3}\right), 2.41 \sim$ $2.54\left(\mathrm{~m}, 10 \mathrm{H}, 5 \times \mathrm{CH}_{2}\right), 3.55\left(\mathrm{~s}, 2 \mathrm{H}, \mathrm{CH}_{2}\right), 7.01(\mathrm{~d}, J=8.0$ $\mathrm{Hz}, 1 \mathrm{H}, \mathrm{Py}-\mathrm{H}), 7.10$ (d, $J=8.0 \mathrm{~Hz}, 2 \mathrm{H}, \mathrm{Ar}-\mathrm{H}), 7.40$ (d, $J=$ $8.0 \mathrm{~Hz}, 2 \mathrm{H}, \mathrm{Ar}-\mathrm{H}), 7.90$ (d, $J=8.0 \mathrm{~Hz}, 1 \mathrm{H}, \mathrm{Py}-\mathrm{H}), 8.46$ (s, $1 \mathrm{H}, \mathrm{Py}-\mathrm{H}) ;{ }^{13} \mathrm{C}$ NMR $\left(100 \mathrm{MHz}, \mathrm{CDCl}_{3}\right) \delta: 12.0,52.3$, 52.8, 53.1, 62.4, 111.3, 121.1, 125.0, 130.5, 135.5, 136.6, 145.4, 145.5, 152.1, 165.9. Anal. calcd for $\mathrm{C}_{19} \mathrm{H}_{22} \mathrm{~F}_{3} \mathrm{~N}_{3} \mathrm{O}: \mathrm{C}$ 62.45, H 6.07, N 11.50; found C 62.63, H 5.89, N 11.32.

1-[4-(5-三氟甲基吡啶-2-基氧基)苯甲基]-4-苯基哌 嗪(5j): 白色固体, 产率 53\%. m.p. 99 101 ${ }^{\circ} \mathrm{C} ;{ }^{1} \mathrm{H}$ NMR $\left(400 \mathrm{MHz}, \mathrm{CDCl}_{3}\right) \delta: 2.71\left(\mathrm{~s}, 4 \mathrm{H}, 2 \times \mathrm{CH}_{2}\right), 3.28(\mathrm{~s}, 4 \mathrm{H}$, $\left.2 \times \mathrm{CH}_{2}\right), 3.65\left(\mathrm{~s}, 2 \mathrm{H}, \mathrm{CH}_{2}\right), 6.92(\mathrm{t}, J=8.0 \mathrm{~Hz}, 1 \mathrm{H}, \mathrm{Ar}-\mathrm{H})$, $7.00(\mathrm{~d}, J=8.0 \mathrm{~Hz}, 2 \mathrm{H}, \operatorname{Ar}-\mathrm{H}), 7.06(\mathrm{~d}, J=8.0 \mathrm{~Hz}, 1 \mathrm{H}$, Py-H), 7.20 (d, $J=8.0 \mathrm{~Hz}, 2 \mathrm{H}, \mathrm{Ar}-\mathrm{H}), 7.33$ (t, $J=8.0 \mathrm{~Hz}$, 2H, Ar-H), 7.50 (d, $J=8.0 \mathrm{~Hz}, 2 \mathrm{H}, \mathrm{Ar}-\mathrm{H}), 7.94$ (d, $J=8.0$ $\mathrm{Hz}, 1 \mathrm{H}, \mathrm{Py}-\mathrm{H}), 8.53$ (s, 1H, Py-H); ${ }^{13} \mathrm{C}$ NMR (100 MHz, $\left.\mathrm{CDCl}_{3}\right) \delta: 49.2,53.2,62.5,111.4,116.1,119.7,121.3$, $125.1,129.2,130.5,135.4,136.7,136.9,145.5,145.6$, 151.4, 152.3, 165.9. Anal. calcd for $\mathrm{C}_{23} \mathrm{H}_{22} \mathrm{~F}_{3} \mathrm{~N}_{3} \mathrm{O}: \mathrm{C} 66.82$, H 5.36, N 10.16; found C 66.98, H 5.21, N 10.02 .

1-[4-(5-三氟甲基吡啶-2-基氧基)苯甲基]-4-芐基哌 嗪(5k): 白色固体, 产率 41\%. m.p. $103 \sim 105{ }^{\circ} \mathrm{C} ;{ }^{1} \mathrm{H}$ NMR (400 MHz, $\left.\mathrm{CDCl}_{3}\right) \delta: 2.47\left(\mathrm{~s}, 6 \mathrm{H}, 3 \times \mathrm{CH}_{2}\right), 3.49(\mathrm{~s}$, $\left.4 \mathrm{H}, 2 \times \mathrm{CH}_{2}\right), 6.95(\mathrm{~d}, J=8.4 \mathrm{~Hz}, 1 \mathrm{H}, \mathrm{Py}-\mathrm{H}), 7.05$ (d, $J=8.4 \mathrm{~Hz}, 2 \mathrm{H}, \mathrm{Ar}-\mathrm{H}), 7.21 \sim 7.28(\mathrm{~m}, 5 \mathrm{H}, \mathrm{Ar}-\mathrm{H}), 7.34$ (d, $J=8.4 \mathrm{~Hz}, 2 \mathrm{H}, \mathrm{Ar}-\mathrm{H}), 7.84$ (d, $J=8.8 \mathrm{~Hz}, 1 \mathrm{H}, \mathrm{Py}-\mathrm{H}), 8.40$ (s, 1H, Py-H); ${ }^{13} \mathrm{C}$ NMR (100 MHz, $\left.\mathrm{CDCl}_{3}\right) \delta: 47.8,57.1$, $57.8,106.0,115.9,119.8,121.8,123.0,124.0,125.2$, $130.2,131.3,131.4,132.8,140.2,140.3,146.9,160.6$; IR (KBr) v: 3082, 3027, 2942, 2874, 2802, 2766, 1615, 1603, 1509, 1485, 1392, 1336, 1258, 1160, 1075, 1011, 847, 741, $698 \mathrm{~cm}^{-1}$. Anal. calcd for $\mathrm{C}_{24} \mathrm{H}_{24} \mathrm{~F}_{3} \mathrm{~N}_{3} \mathrm{O}$ : C 67.43, H 5.66, N 9.83; found C 67.59, H 5.51, N 9.65.

1-[4-(5-三氟甲基吡啶-2-基氧基)苯甲基]吗啉(5l): 白色固体，产率 50\%. m.p. 44 46 ${ }^{\circ} \mathrm{C} ;{ }^{1} \mathrm{H}$ NMR (400
$\left.\mathrm{MHz}, \mathrm{CDCl}_{3}\right) \delta: 2.48\left(\mathrm{t}, J=4.4 \mathrm{~Hz}, 4 \mathrm{H}, 2 \times \mathrm{CH}_{2}\right), 3.52(\mathrm{~s}$, $\left.2 \mathrm{H}, \mathrm{CH}_{2}\right), 3.72\left(\mathrm{t}, J=4.8 \mathrm{~Hz}, 4 \mathrm{H}, 2 \times \mathrm{CH}_{2}\right), 7.00(\mathrm{~d}, J=8.4$ $\mathrm{Hz}, 1 \mathrm{H}, \mathrm{Py}-\mathrm{H}), 7.10$ (d, $J=8.4 \mathrm{~Hz}, 2 \mathrm{H}, \mathrm{Ar}-\mathrm{H}), 7.39$ (d, $J=$ $8.4 \mathrm{~Hz}, 2 \mathrm{H}, \mathrm{Ar}-\mathrm{H}), 7.88$ (d, $J=8.8 \mathrm{~Hz}, 1 \mathrm{H}, \mathrm{Py}-\mathrm{H}$ ), 8.44 (s, $1 \mathrm{H}, \mathrm{Py}-\mathrm{H}) ;{ }^{13} \mathrm{C}$ NMR $\left(100 \mathrm{MHz}, \mathrm{CDCl}_{3}\right) \delta: 53.6,62.8$, 67.0, 111.3, 121.2, 130.5, 135.1, 136.6, 136.7, 145.4, 145.5, 152.2, 165.8. Anal. calcd for $\mathrm{C}_{17} \mathrm{H}_{17} \mathrm{~F}_{3} \mathrm{~N}_{2} \mathrm{O}_{2}$ : $\mathrm{C}$ 60.35, H 5.06, N 8.28; found C 60.51, H 4.92, N 8.46.

1-[4-(5-三氟甲基吡啶-2-基氧基)苯甲基]哌啶 $(\mathbf{5 m})$ : 白色固体，产率 52\%. m.p. 45 47 ${ }^{\circ} \mathrm{C} ;{ }^{1} \mathrm{H}$ NMR (400 $\left.\mathrm{MHz}, \mathrm{CDCl}_{3}\right) \delta: 1.36 \sim 2.31\left(\mathrm{~m}, 10 \mathrm{H}, 5 \times \mathrm{CH}_{2}\right), 3.39(\mathrm{~s}$, $2 \mathrm{H}, \mathrm{CH}_{2}$ ), 6.90 (d, $\left.J=8.4 \mathrm{~Hz}, 1 \mathrm{H}, \mathrm{Py}-\mathrm{H}\right), 7.00$ (d, $J=8.4$ $\mathrm{Hz}, 2 \mathrm{H}, \mathrm{Ar}-\mathrm{H}), 7.29$ (d, $J=8.8 \mathrm{~Hz}, 2 \mathrm{H}, \mathrm{Ar}-\mathrm{H}), 7.79$ (d, $J=$ $8.8 \mathrm{~Hz}, 2 \mathrm{H}, \mathrm{Py}-\mathrm{H}), 8.36$ (s, 1H, Py-H); ${ }^{13} \mathrm{C}$ NMR (100 $\left.\mathrm{MHz}, \mathrm{CDCl}_{3}\right) \delta: 23.4,25.0,53.5,62.2,110.2,120.0,129.5$, $135.0,135.5,135.6,144.4,144.5,151.0,164.9$. Anal. calcd for $\mathrm{C}_{18} \mathrm{H}_{19} \mathrm{~F}_{3} \mathrm{~N}_{2} \mathrm{O}$ : C 64.28, H 5.69, $\mathrm{N} 8.33$; found $\mathrm{C}$ 64.11, H 5.83, N 8.50.

\section{4 生物活性测试}

杀虫活性测试所用昆虫分别为: 小菜蛾 (Plutella xylostella)、蚜虫 (Aphis craccivora) 、朱砂叶螨 (Tetranychus cinnabarinus)和褐飞虫(Nilaparvata lugens). 对照药剂分别为: 啶虫丙醚 (Pyridalyl)、吡虫啉 (Imidacloprid)和唑螨酯(Fenpyroximate).

药剂配置: 对于化合物, 用分析天平称取一定质量 的原药, 用含吐温-80 乳化剂的 DMF 溶解配制成 $1 \%$ 母 液, 然后用蒸馏水稀释即得到 $200 \mu \mathrm{g} / \mathrm{mL}$ 的药液, 其它 浓度的药液可由此进行稀释. 每个处理 3 次重复, 设空 白对照.

小菜蛾: 采用浸叶碟法. 首先, 用直头眼科镊子浸渍 甘蓝叶片, 时间 $2 \sim 3 \mathrm{~s}$, 甩掉余液. 每次 1 片, 每个样品 共 3 片. 按照样品标记顺序放在处理纸上, 待药液干后, 放入具有标记的 $10 \mathrm{~cm}$ 长的直型管内, 接入 2 龄小菜蛾 幼虫, 用纱布盖好管口. 将试验处理置于标准处理室内, 查药后 $2 \mathrm{~d}$.

辅助材料(Supporting Information) 化合物 $\mathbf{5 a} \sim 5 \mathrm{~m}$ 的 ${ }^{1} \mathrm{H}$ NMR 和 ${ }^{13} \mathrm{C}$ NMR 图谱. 这些材料可以免费从本刊网 站(http://sioc-journal.cn/)上下载.

\section{References}

[1] Sumida, M.; Niwata, S.; Fukami, H.; Tanaka, T.; Wakabayashi, K.; Boger, P. J. Agric. Food Chem. 1995, 43, 1929.

[2] Wang, M. H.; Yang, C. L.; Jiang, M. G. World Pestic. 2002, 24, 13 (in Chinese).

(王鸣华, 杨春龙，蒋木庚，世界农药, 2002, 24, 13.) 
[3] Ning, L. H.; Peng, H.; Tu, H. Y.; He, H. W. Chin. J. Pestic. Sci. 2004, 6, 74 (in Chinese). (宁丽红, 彭浩, 涂海洋, 贺红武, 农药学学报, 2004, 6, 74.)

[4] Freundlich, J. S.; Yu, M.; Lucumi, E.; Kuo, M.; Tsai, H. C.; Valderramos, J. C.; Karagyozov, L.; Jacobs, W. R. Jr.; Schiehser, G. A.; Fidock, D. A.; Jacobus, D. P.; Sacchettini, J. C. Bioorg. Med. Chem. Lett. 2006, 16, 2163.

[5] Liu, A. P.; Feng, Q. J.; Hu, C. H.; He, H. J.; Wang, T. J.; Liu, Y.; Lei, M. X.; Pang, H. L.; He, H. W. Chin. J. Org. Chem. 2008, 28, 531 (in Chinese).

(柳爱平, 冯秋菊, 胡春华, 何海军, 王婷瑾, 刘洋, 雷满香, 庞 怀林, 贺红武, 有机化学, 2008, 28, 531.)

[6] Kini, S. G.; Bhat, A. R.; Byrant, B.; Williamson, J. S.; Dayan, F. E. Eur. J. Med. Chem. 2009, 44, 492.

[7] Liu, J. C.; Cui, Z. P.; He, H. W. Chin. J. Org. Chem. 2012, 32, 1925 (in Chinese). (刘建超, 崔泽平, 贺红武, 有机化学, 2012, 32, 1925.)

[8] Anderson, J. W.; Sarantakis, D.; Terpinski, J.; Kumar, T. R. S.; Tsai, H. C.; Kuo, M.; Ager, A. L.; Jacobs, W. R. Jr.; Schiehser, G. A.; Ekins, S.; Sacchettini, J. C.; Jacobus, D. P.; Fidock, D. A.; Freundlich, J. S. Bioorg. Med. Chem. Lett. 2013, 23, 1022.

[9] Peng, H.; Gao, Y. J.; He, H. W. Chin. J. Org. Chem. 2013, 33, 1994 (in Chinese). (彭浩, 高玉焦, 贺红武, 有机化学, 2013, 33, 1994.)

[10] Zhang, Y. P.; Tan, W.; Yang, Y. S.; Sun, S. Q.; Guo, H. C. Chin. J. Org. Chem. 2015, 35, 1985 (in Chinese). (张应鹏, 谭伟, 杨云裳, 孙世琪, 郭慧琛, 有机化学, 2015, 35, 1985.)

[11] Kleemann, A.; Baltruschat, H. S.; Huelsen, T.; Thomas, S. S. EP 0723960, 1996 [Chem. Abstr. 1996, 125, 195679].

[12] Morimoto, K.; Ohnari, M.; Furusawa, H.; Terachi, T.; Nawamaki, T.; Ishikawa, K.; Nakahira, K.; Kawaguchi, C. WO 9606096, 1996 [Chem. Abstr. 1996, 125, 58524].

[13] Xu, Y. J.; Wang, Q. H.; Wu, J. Y.; Wu, H. B.; Ran, Z. J.; Mao, C. S.; Qin, Z. H. Chin. J. Pestic. Sci. 2007, 9, 189 (in Chinese). (徐彦军, 王庆海, 武菊英, 吴厚斌, 冉兆晋, 毛朝姝, 覃兆海, 农药学学报, 2007, 9, 189.)

[14] Ran, Z. J.; Ni, H. W.; Duan, H. X.; Li, N.; Dong, Y. H.; Fu, B.; Xiao, Y. M.; Qin, Z. H. Chin. J. Pestic. Sci. 2009, 11, 41 (in Chinese).

(再兆晋, 倪汉文, 段红霞, 李楠, 董燕红, 傅滨, 肖玉梅, 覃兆
海, 农药学学报, 2009, 11, 41.)

[15] Li, R. J.; Bai, Y. H.; Shi, B.; Xiao, P. F. Chin. J. Pestic. 2007, 46, 305 (in Chinese).

(李瑞军, 白延海, 史坢, 肖鹏飞, 农药, 2007, 46, 305.)

[16] Li, A. J.; Liu, B. S.; Yuan, B. G. Chin. J. Pestic. 2012, 51, 416 (in Chinese).

(李爱军, 刘宝擅, 苑保国, 农药, 2012, 51, 416.)

[17] Ran, Z. J.; Yuan, H. Z.; Cao, A. C.; Qin, Z. H. Chin. J. Pestic. Sci. 2010, 12, 269 (in Chinese).

(再兆晋, 袁会珠, 曹坳程, 覃兆海, 农药学学报, 2010, 12, 269.)

[18] Ran, Z. J.; Yuan, H. Z.; Cao, A. C.; Qin, Z. H. Chin. J. Pestic. Sci. 2010, 12, 458 (in Chinese).

(虫兆晋, 袁会珠, 曹坳程, 覃兆海, 农药学学报, 2010, 12, 458.)

[19] Zhang, A. G.; Kayser, H.; Maienfisch, P.; Casida, J. E. J. Neurochem. 2000, 75, 1294.

[20] Zhang, G. S.; Hou, G. X. Pestic. Sci. Admin. 2004, 25, 22 (in Chinese).

(张国生, 侯广新, 农药科学与管理, 2004, 25, 22.)

[21] Dai, B. J. World Pestic. 2005, 27, 46 (in Chinese). (戴宝江, 世界农药, 2005, 27, 46.)

[22] Shao, X. S.; Tian, Z. Z.; Li, Z.; Xu, X. Y.; Huang, Q. C.; Qian, X. H. Chin. J. Pestic. Sci.. 2008, 10, 117 (in Chinese). (邵旭升, 田忠贞, 李忠, 徐晓勇, 黄青春, 钱旭红, 农药学学报, 2008, 10, 117.)

[23] Yokota, T.; Mikata, K.; Nagasaki, H.; Ohta, K. J. Agric. Food Chem. 2003, 51, 7066 .

[24] Kagabu, S.; Ishihara, R.; Hieda, Y.; Nishimura, K.; Naruse, Y. J. Agric. Food Chem. 2007, 55, 812.

[25] Bouillot, A. M. J.; Dodic, N.; Gellibet, F. J.; Mirguet, O. WO 2009071504, 2009 [Chem. Abstr. 2009, 151, 33581].

[26] Wang, T. T.; Bing, G. F.; Zhang, X.; Qin, Z. F.; Yu, H. B.; Qin, X.; Dai, H.; Miao, W. K.; Wu, S. S.; Fang, J. X. Bioorg. Med. Chem. Lett. 2010, 20, 3348.

[27] Wu, S. S.; Miao, W. K.; Wang, T. T.; Fang, J. X. Chin. J. Org. Chem. 2015, 35, 1484 (in Chinese). (吴珊珊, 苗文科, 王婷婷, 方建新, 有机化学, 2015, 35, 1484.)

[28] Ma, Y. M.S. Thesis, Nankai University, Tianjin, 2009 (in Chinese). (马云, 硕士论文, 南开大学, 天津, 2009.)

[29] Liu, J. C.; Liu, Y. J.; He, H. W. Chin. J. Org. Chem. 2015, 35, 462 (in Chinese).

(刘建超, 刘勇军, 贺红武, 有机化学, 2015, 35, 462.) 